Pesq. Vet. Bras. 30(3):191-194, março 2010

\title{
Intoxicação por Trema micrantha (Ulmaceae) em caprinos no Estado de Santa Catarina ${ }^{1}$
}

\author{
Aldo Gava ${ }^{2}$, Joelma Lucioli ${ }^{3}$, Fernando Henrique Furlan³ ${ }^{3}$ Maurício Buss Leal ${ }^{4}$ \\ e Sandra Davi Traverso2*
}

\begin{abstract}
Gava A., Lucioli J., Furlan F.H., Leal M.B. \& Traverso S.D. 2010. [Poisoning by Trema micrantha (UImaceae) in goats in the State of Santa Catarina.] Intoxicação por Trema micrantha (Ulmaceae) em caprinos no Estado de Santa Catarina. Pesquisa Veterinária Brasileira 30(3):191-194. Departamento de Medicina Veterinária, Centro de Ciências Agroveterinárias, Universidade do Estado de Santa Catarina, Lages, SC 88520000, Brazil. E-mail: a2sdt@cav.udesc.br

Twenty five goats, maintained in paddocks, had died in five subsequent days after have been offered leaves, mixed in the ration, of Trema micrantha, a tree commonly called grandiúva. Four animals were necropsied and samples were collected for histopathology. Clinical signs included apathy, anorexia, head pressing against obstacles, decubitus and death. Macroscopic findings included suffusions in the epi- and endocardium, a yellowish liver with pronounced lobular pattern, in one goat, the liver presented additionally multiple visible hemorrhages. Histological examination revealed centrilobular to massive hepatic necrosis consistent with acute liver toxicosis. In the brain, satelitosis, neuronal swelling, and perineuronal and perivascular edema was found. The diagnosis of poisoning by Trema micrantha was based in the clinical picture characteristic of toxic hepatitis associated in the feeding of the plant to the goats.
\end{abstract}

INDEX TERMS: Poisonous plants, Trema micrantha, Ulmaceae, grandiúva, plant poisoning, hepatotoxic plants, goats.

RESUMO.- Vinte e cinco caprinos, criados em piquetes, morreram nos cinco dias subsequentes ao fornecimento de folhas de Trema micrantha (fam.Ulmaceae), uma árvore com nome comum de grandiúva. Quatro caprinos foram necropsiados e amostras de vísceras foram coletadas para exame histológico. As principais alterações clínicas foram: apatia, anorexia, cabeça apoiada contra obstáculos, decúbito e morte. Achados macroscópicos incluíram sufusões no epicárdio e endocárdio; fígado levemente amarelado e com padrão lobular evidente e, em um caso, acompanhado de hemorragias multifocais. Na histologia observou-se necrose hepática, que variava de centrolobular a massiva,

\footnotetext{
${ }^{1}$ Recebido em 15 de abril de 2009.

Aceito para publicação em 19 de setembro de 2009.

2 Departamento de Ciê-ncia e Patologia, CAV, Universidade do Estado de Santa Catarina (UDESC), Av. Luis de Camões 2090, Lages, SC 88520-000, Brasil. *Autor para correspondência: a2sdt@ cav.udesc.br

3 Programa de Pós-Graduação em Ciências Veterinárias, Área de concentração em Patologia Veterinária, CAV/UDESC, Lages, SC.

${ }^{4}$ Médico Veterinário Autônomo, Rua Anita Garibaldi 297, Apto 122, Itajaí, SC 88330-020, Brasil.
}

compatível com hepatopatia tóxica. No SNC havia satelitose, tumefação neuronal, edema periaxonal, perivascular. O diagnóstico de intoxicação por Trema micrantha foi baseado no quadro clínico e lesional de hepatite tóxica associado ao uso da planta para alimentação de caprinos.

TERMOS DE INDEXAÇÃO: Plantas tóxicas, Trema micrantha, Ulmaceae, grandiúva, intoxicação por planta, planta hepatotóxica, caprinos.

\section{INTRODUÇÃO}

Um surto de mortalidade em caprinos com quadro clínico e lesional de insuficiência hepática aguda ocorreu no município de Navegantes, SC. As mortes ocorreram após o fornecimento de folhas de uma árvore conhecida na região por grandiúva (Trema micrantha). No Estado de Santa Catarina são frequentes as informações de produtores que dizem ter utilizado folhas de "grandiúva" para alimentação de bovinos. Há também informações referentes à utilização dessa planta para coelhos, porém para essa espécie, parece haver conhecimento por parte de criadores sobre a possível toxicidade das folhas de grandiúva. 


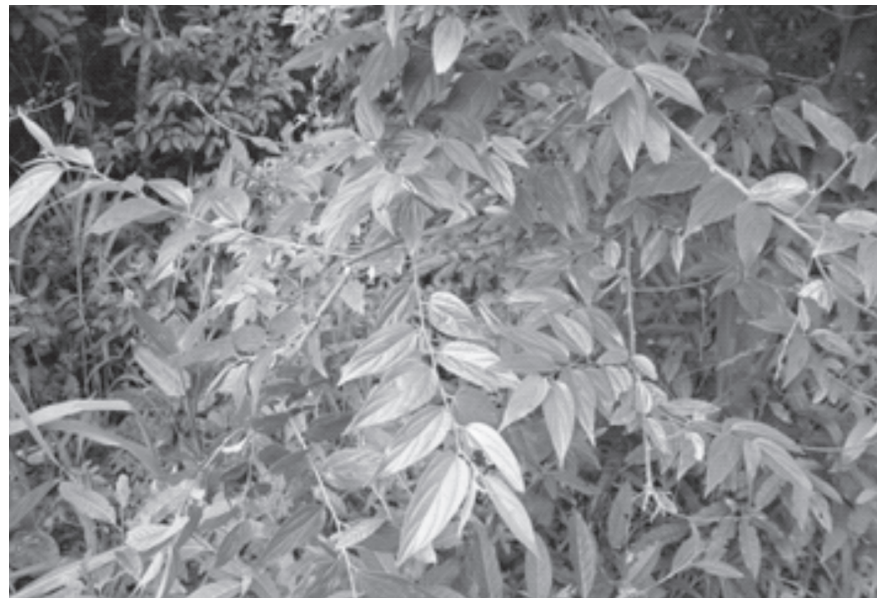

Fig.1. Trema micrantha L. em Santa Catarina.

Trema micrantha L. (Fig.1), da família Ulmaceae, é encontrada em todo Brasil e conhecida vulgarmente por grandiúva, pau-pólvora ou periquiteiro (Lorenzi 1992). Intoxicação espontânea por folhas desta árvore foi descrita em caprinos no Rio Grande do Sul (Traverso et al. 2003, 2005) e intoxicação experimental foi descrita em coelhos (Traverso \& Driemeier 2000), cabras (Traverso et al. 2002) e bovinos (Traverso et al. 2004). O presente estudo tem como objetivo relatar o primeiro surto de intoxicação natural por T. micrantha ocorrida no estado de Santa Catarina.

\section{MATERIAL E MÉTODOS}

Dados epidemiológicos de uma enfermidade em caprinos, ocorrida no Município de Navegantes, SC, foram obtidos com o veterinário responsável. Animais doentes foram necropsiados e amostras de tecidos foram coletadas, fixadas em formol $10 \%$, processadas para exame histológico e coradas pela técnica de hematoxilina e eosina $(\mathrm{HE})$.

\section{RESULTADOS}

Em uma criação de caprinos Boer, localizada no município de Navegantes, litoral de Santa Catarina, um lote de 120 caprinos mantidos em piquetes com Brachiaria decumbens e alimentados no cocho com cana triturada e casquinha de soja, receberam adicionalmente folhas de Trema micrantha por um período de dois dias. No segundo dia após o fornecimento da planta, uma cabra morreu. Nos cinco dias subsequentes 24 animais apresentaram perda de apetite, apatia, atonia ruminal, fezes em pequena quantidade e recobertas por muco. Alguns animais apoiavam a cabeça contra a parede. Todos os caprinos doentes morreram.

A necropsia dos quatro caprinos revelou sufusões no epicárdio e endocárdio, fígado amarelado com evidenciação do padrão lobular (Fig.2) e, em um caprino, associado a hemorragias de 0,1-0,5mm de diâmetro visíveis na superfície capsular e de corte, edema de vesícula biliar e conteúdo do cólon e reto envolto por muco e estrias de sangue.

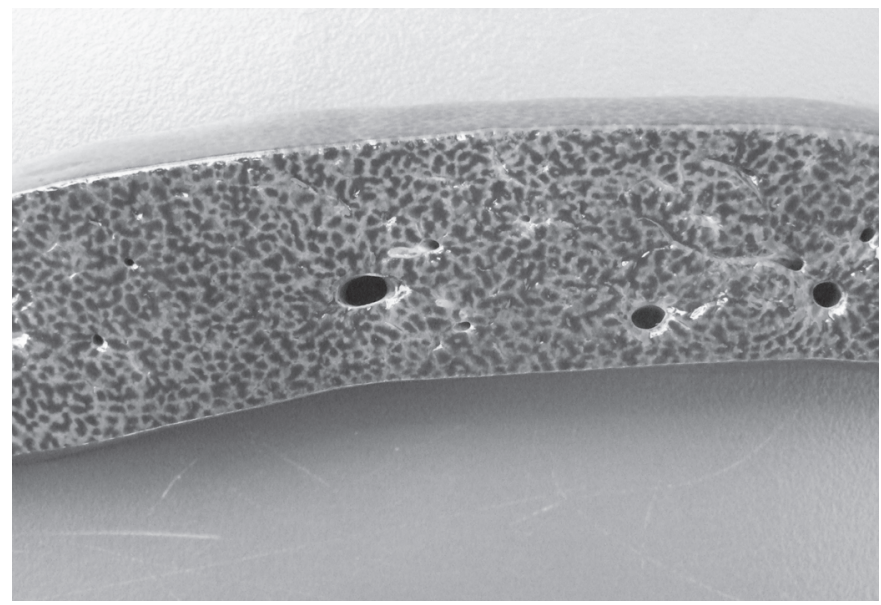

Fig.2. Fígado de caprino intoxicado espontaneamente por Trema micrantha com padrão lobular evidente.

Ao exame histológico observou-se, no fígado de dois caprinos, necrose massiva de hepatócitos acompanhada de congestão e hemorragia centrolobular (Fig.3). Nos dois outros caprinos a necrose hepática foi centrolobular com degeneração vacuolar no citoplasma dos hepatócitos da periferia dos lóbulos. Somente dois encéfalos foram analisados e neles havia tumefação neuronal, principalmente de córtex, satelitose, edema perineuronal, perivascular e na camada molecular do cerebelo; em um deles havia vacuolização da substância branca do cerebelo.

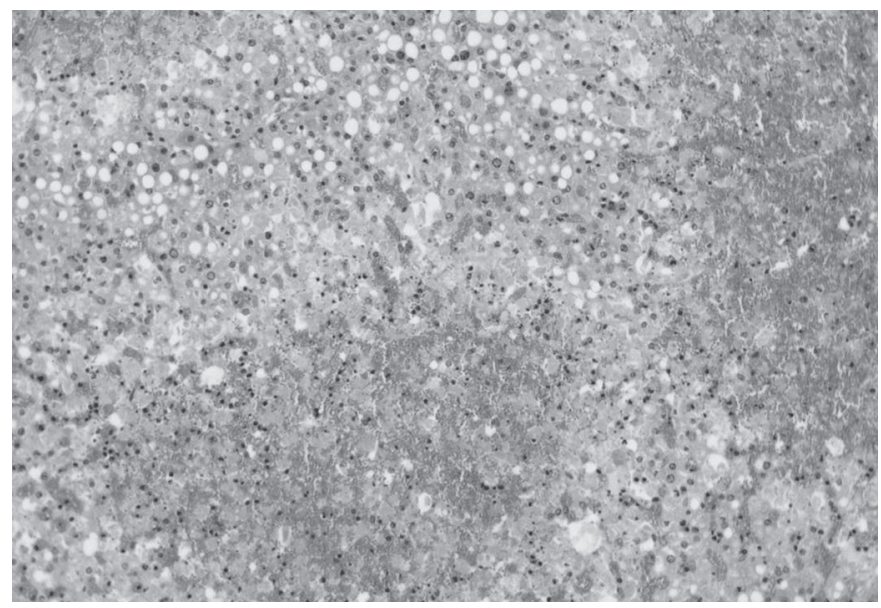

Fig.3. Necrose e congestão centrolobular cercada por degeneração vacuolar de hepatócitos, em fígado de caprino intoxicado espontaneamente por Trema micrantha. HE, obj.20x.

\section{DISCUSSÃO}

O quadro clínico e lesional observado nesse surto é semelhante aos descrito na intoxicação natural (Traverso et al. 2003, 2005) e experimental por Trema micrantha em caprinos (Traverso et al. 2002).

$\mathrm{Na}$ intoxicação experimental por T. micrantha em caprinos o período relatado entre a ingestão da planta e a morte do animal variava de 86 a 123 horas após a ingestão da planta (Traverso et al. 2002). No surto em questão os 
animais morreram entre dois e cinco dias após a ingestão da planta, o que se assemelha ao observado experimentalmente. O período prolongado entre a ingestão da planta e a morte dos animais é um fator epidemiológico importante na intoxicação por T. micrantha, pois ela difere das demais plantas de ação hepatotóxica aguda. Na intoxicação experimental aguda em caprinos por Vernonia mollissima, a morte ocorre até 24 horas após a administração da planta (Stolf et al. 1987); da mesma forma, plantas que causam necrose hepática aguda em bovinos, como Sessea brasiliense (Canella et al. 1968), Cestrum laevigatum (Döbereiner et al. 1969), C. intermedium (Gava et al. 1996), Xanthium cavanillensii (Driemeier et al. 1999, Colodel et al. 2000) e Dodonea viscosa (Cattani et al. 2004), o tempo de morte, de maior período relatado, é de 55 horas após a ingestão da planta.

A alteração hepática observada nesse surto é compatível com intoxicação por plantas de ação hepatotóxica aguda e foi caracterizada por necrose de coagulação de hepatócitos que variavam de centrolobular a massiva. As alterações histológicas no fígado, observadas nas intoxicações por plantas que causam necrose hepática aguda são semelhantes entre si e muitas dessas lesões, são as mesmas para diferentes plantas. A melhor maneira para chegar à conclusão de qual planta está envolvida no processo, é sempre a constatação da planta no local, embora algumas alterações histológicas possam sugerir qual planta pode estar envolvida na mortalidade.

$\mathrm{Na}$ intoxicação por Sessea brasiliense, a necrose é centrolobular e associada à vacuolização dos hepatócitos adjacentes (Canella et al. 1968). Nas plantas do gênero Cestrum, a intensidade da necrose é variável, sendo centrolobular com vacuolização dos hepatócitos adjacentes nos casos de intoxicação por $C$. laevigatum (Döbereiner et al. 1969), C. parqui (Riet-Correa et al. 1986) e necrose centrolobular ou atingindo todo o lóbulo, na intoxicação por $C$. corymbosum (Gava et al. 1991) e C. intermedium (Gava et.al. 1996, Furlan et al. 2008). Na intoxicação por Vernonia spp. há, na grande maioria dos casos, necrose e lise de quase todo o parênquima, restando poucas células hepáticas na periferia dos lóbulos (Tokarnia et al. 2000). Nas intoxicações por Dodonea viscosa (Colodel et al. 2003, Cattani et al. 2004), Xanthium (Driemeier et al. 1999, Colodel et al. 2000) e Trema micrantha (Traverso et al. 2002, 2004), a necrose varia de centrolobular a massiva, acompanhada, na necrose centrolobular, de vacuolização de hepatócitos na periferia.

Além das alterações histológicas hepáticas, as alterações extra-hepáticas podem auxiliar na etiologia da planta envolvida. Nas intoxicações por Trema micrantha (Traverso et al. 2002, 2004) e plantas do gênero Vernonia spp. (Tokarnia \& Döbereiner 1982, Tokarnia et al. 1986, Gava et al. 1987, Stolf et al. 1987, Brum et al. 2002) degeneração tubular renal pode ser observada. No caso específico de Trema micrantha, a degeneração neuronal é bastante sugestiva quando associada à lesão hepática; nesses casos as alterações nervosas localizam-se na substância cinzenta do encéfalo, diferenciando-se das demais espécies de plantas hepatotóxicas que não apresentam lesão, ou no caso de Sessea brasiliensis, são caracterizadas por vacuolização de substância branca (Chaulet et al. 1990).

Nesse estudo, as lesões histológicas observadas no sistema nervoso central foram semelhantes àquelas relatadas nas intoxicações espontâneas e experimental por T. micrantha em caprinos (Traverso et al. 2002, 2003), e na intoxicação experimental em bovinos (Traverso et al. 2004), e provavelmente se devam a hipoglicemia que foi constatada na intoxicação experimental por $T$. micrantha em caprinos (Traverso et al. 2002). Além de lesões corticais, foi observada também, em um caprino, vacuolização de substância branca, o que ainda não havia sido relatado na intoxicação por Trema, indicando que a hiperamonemia também deva ocorrer durante a intoxicação. Lesões renais não foram observadas.

No Brasil, relatos de intoxicação espontânea em caprinos por outras plantas hepatotóxicas como Cestrum laevigatun foi realizada por Peixoto et al. (2000), e intoxicações experimentais foram descritas com Sessea brasiliensis (Chaulet et al. 1990) e Vernonia mollissima (Stolf et al. 1987), fatos a serem considerados no diagnóstico diferencial. Além dessas plantas, no Estado de Santa Catarina, nos casos de necrose hepática aguda, intoxicações por $C$. intermedium, C. corymbosum e Xanthium sp. também devem ser consideradas, pois são as plantas frequentemente associadas à mortalidades de bovinos com hepatopatia tóxica aguda (Borelli et al. 2008).

\section{CONCLUSÃO}

A enfermidade de caprinos ocorrida no município de Navegantes, SC, caracterizou-se por necrose hepática aguda. O diagnóstico de intoxicação por Trema micrantha, neste surto, foi baseado no relato do fornecimento da planta aos animais e na semelhança entre as lesões observadas com aquelas relatadas nos outros casos/surtos de intoxicação por T. micrantha.

\section{REFERÊNCIAS}

Borelli V., Zandonai A.D., Furlan F.H., Traverso S.D. \& Gava A. 2008. Intoxicação por plantas diagnosticadas em bovinos pelo laboratório de Patologia Animal, CAV/UDESC, de janeiro de 2000 a abril de 2008. Anais Encontro Nacional de Diagnóstico Veterinário, (Endivet), Campo Grande, MS. CD-ROM.

Brum K.B., Purisco E., Lemos R.A.A. \& Riet-Correa F. 2002. Intoxicação por Vernonia rubricaulis em bovinos no Mato Grosso do Sul. Pesq. Vet. Bras. 22(3):147-148.

Canella C.F.C., Tokarnia C.H. \& Döbereiner J. 1968. Intoxicação por Sessea brasiliensis Toledo em bovinos. Pesq. Agropec. Bras, Sér. Vet., 3:333-340.

Cattani C.S., Colodel E.M., Traverso S.D., Correa A.M.R. \& Driemeier D. 2004. Intoxicação experimental por Dodonea viscosa (Sapindaceae) em bovinos. Pesq. Vet. Bras. 24(1):31-34.

Chaulet J.H.F., Peixoto P.V. \& Tokarnia C.H. 1990. Intoxicação experimental por Sessea brasiliensis (Solanaceae) em bovinos ovinos e caprinos. Pesq. Vet. Bras. 10(3/4):71-84. 
Colodel E.M., Driemeier D. \& Pilati C. 2000. Intoxicação experimental pelos frutos de Xanthium cavanillesii (Asteraceae) em bovinos. Pesq. Vet. Bras. 20(1):31-38.

Colodel E.M., Traverso S.D., Seitz A.L., Oliveira F.N., Driemeier D. \& Gava A. 2003. Spontaneous poisoning by Dodonea viscosa (Sapindaceae) in cattle. Vet. Human Toxicol. 45(3):147-148.

Döbereiner J., Tokarnia C.H. \& Canella C.F.C. 1969. Intoxicação por Cestrum laevigatum Schlecht., a causa de mortandades em bovinos no Estado do Rio de Janeiro. Pesq. Agropec. Bras.4:165193.

Driemeier D., Irigoyen L.F., Loretti A.P., Colodel E.M. \& Barros C.S.L 1999. Intoxicação espontânea pelos frutos de Xanthiun cavanillesii (Asteraceae) em bovinos no Rio Grande do Sul. Pesq.Vet. Bras. 19(1):1-13.

Furlan F.H., Lucioli J., Borelli V., Faria O., Rebelatto S.V., Gava A. \& Traverso S.D. 2008. Intoxicação por Cestrum intermedium (Solanaceae) em bovinos. Acta Scientiae Veterinariae 36:281-284.

Gava A., Peixoto P.V. \& Tokarnia C.H. 1987. Intoxicação experimental por Vernonia mollissima em ovinos e bovinos. Pesq. Vet. Bras. 7(2):3341.

Gava A., Stolf L., Pilati C., Neves D.S. \& Viganó L. 1991. Intoxicação por Cestrum corymbosum var. hirsutum (Solanaceae) em bovinos no Estado de Santa Catarina. Pesq. Vet. Bras. 11(3/4):71-74.

Gava A., Stolf L., Varaschin M.S., Neves D.S., Tigre A.P. \& Lessmann F. 1996. Intoxicação por Cestrum intermedium (Solanaceae) em bovinos. Pesq. Vet. Bras. 16(4):117-120.

Lorenzi H. 1992. Árvores Brasileiras: manual de identificação e cultivo de plantas arbóreas ativas do Brasil. Editora Platarum, Nova Odessa, p.340-341.

Peixoto P.V., Brust L.A.C., Brito M.F., França T.N., Cunha B.R.M. \&
Andrade G.B. 2000. Cestrun laevigatum poisoning in goats in southeastern Brazil. Vet. Hum. Toxicol. 42(1):13-14.

Riet-Correa F., Schild A.L., Mendez M.C. \& Pinheiro M. 1986. Intoxicação por Cestrum parqui (Solaneae) em bovinos no Rio Grande do Sul. Pesq. Vet. Bras. 6(4):111-115.

Stolf L., Gava A. \& Tokarnia C.H. 1987. Intoxicação experimental por Vernonia mollissima (Compositae) em caprinos. Pesq. Vet. Bras. 7(3):67-77.

Tokarnia C.H. \& Döbereiner J. 1982. Intoxicação de bovinos por Vernonia rubricaulis (Compositae) em Mato Grosso. Pesq. Vet. Bras. 2(4):143-147.

Tokarnia C.H., Döbereiner J., Amorin P.R., Gava A. \& Consorte L.B. 1986. Intoxicação experimental por Vernonia mollissima (Compositae) em coelhos. Pesq. Vet. Bras. 6(2):5-10.

Tokarnia C.H., Döbereiner J. \& Peixoto P.V. 2000. Plantas Tóxicas do Brasil. Editora Helianthus, Rio de Janeiro, p.91-96.

Traverso S.D. \& Driemeier D. 2000. Experimental Trema micrantha (Ulmaceae) poisoning in rabbits. Vet. Hum. Toxicol. 42(5):301-302.

Traverso S.D., Correa A.M.R., Pescador C.A., Colodel E.M., Cruz C.E.F. \& Driemeier D. 2002. Intoxicação experimental por Trema micrantha (Ulmaceae) em caprinos. Pesq. Vet. Bras. 22(4):141-147.

Traverso S.D., Colodel E.M., Loretti A.P., Correa A.M.R. \& Driemeier D. 2003. Intoxicação natural por Trema micrantha em caprinos. Ciência Rural, 33(1):01-03.

Traverso S.D., Corrêa A.M.R., Schmitz M., Colodel E.M. \& Driemeier D. 2004. Intoxicação experimental por Trema micrantha (Ulmaceae) em bovinos. Pesq Vet Bras. 24(4):211-216.

Traverso S.D., Zlotowski P., Germer M., Cruz C.E.F. \& Driemeier D. 2005. Spontaneous poisoning by Trema micrantha (Ulmaceae) in goats. Acta Scientiae Veterinariae 33(22):207-210. 